\title{
CMOS Pixel Development for the ATLAS Experiment at HL-LHC
}

\author{
B. Ristic ${ }^{1,2}$ on behalf of the ATLAS Collaboration \\ 1 CERN, 1211 Genève 23, Switzerland \\ 2 Physics Department, Lancaster University \\ Lancaster, LA1 4YB, United Kingdom \\ bristic@cern.ch
}

\begin{abstract}
The high luminosity upgrade of the LHC necessitates a complete exchange of the current ATLAS Inner Tracker for a purely silicon one. Large areas therefore have to be covered by radiation tolerant, low cost and low material budget silicon detectors. New approaches are being explored using CMOS pixel sensors, providing charge collection in a depleted layer. They are based on technologies that allow to use high depletion voltages and high resistivity wafers for large depletion depths with multiple nested wells, enabling for CMOS electronics to be embedded safely into the sensor substrate. We are investigating depleted CMOS pixel detectors with monolithic or hybrid designs in view of their suitability for high trigger rates, fast timing and the high radiation environment at HL-LHC. This paper will discuss recent results of the main candidate technologies and the current developments towards a monolithic solution.
\end{abstract}

Keywords: ATLAS ITk, CMOS Pixel Sensors, HV-CMOS, HR-CMOS, CCPD, DMAPS, radiation hardness

\section{Introduction}

Around 2025, the LHC will undergo a major upgrade by which the instantaneous luminosity will be increased by an order of magnitude. The expected radiation levels for the innermost detector will lead to an end-of-life Total Ionizing Dose (TID) up to $1.7 \mathrm{Grad}$ (17 MGy) and Non Ionizing Energy Loss (NIEL) fluences up to $2 \cdot 10^{16} \mathrm{n}_{\mathrm{eq}} \mathrm{cm}^{-2}$. Combined with the increased occupancy and pileup, a complete exchange of the Inner Detector for a purely silicon one becomes necessary. While radiation effects dominate the design of the inner layers of the upgraded Pixel Detector, the outermost ones are limited by construction cost. Pixel sensors in CMOS technology offer several possibilities to reduce cost by producing sensors in industry standard processes and allowing for cheap hybridisation techniques.

While CMOS based sensors, known as Monolithic Active Pixel Sensors (MAPS) are well known in the HEP community (e.g. AIDA beam telescopes [1]), the 
key element for radiation hardness, drift-based charge collection, could only be achieved recently by exploiting High Voltage or High Resistivity CMOS (HV/HR-CMOS) processes. HV-CMOS processes allow for supplying a high bias voltage of over $100 \mathrm{~V}$ to the charge collecting diode while isolating low voltage electronics, implemented on the same substrate. Additionally, HR-CMOS processes use substrates with a resistivity of up to several $\mathrm{k} \Omega \mathrm{cm}$ compared to several 10 to $100 \Omega \mathrm{cm}$ of other CMOS technologies. In both cases, deep implants shield the LV electronics that is implemented on their surface and can simultaneously act as the collecting diode. The general structure of the pixel cell, shown in Figure 1a, depends on the amount of the pixel cell area which is covered by the collecting electrode, called fill factor. A small fill factor electrode leads to a decreased capacitance of the diode, thus a higher and faster output signal at the expense of a less uniform electric field. This in turn necessitates special techniques to ensure that the depletion zone reaches into the space between the electrodes for efficient charge collection after irradiation [2].

The pixel cell usually comprises a multi-stage Charge Sensitive Amplifier (CSA) and discriminator. Output signals have to be further processed by dedicated readout electronics, in this paper the ATLAS IBL FE-I4 chip, to which the sensors are coupled by gluing, thus forming a Capacitively Coupled Pixel Device $(\mathrm{CCPD})$ or by implementing the necessary circuits on the same substrate resulting in a monolithic detector.

After an investigation of prototypes from different foundries, we now focus on the AMS (a)H18 $180 \mathrm{~nm} / \mathrm{H} 35350 \mathrm{~nm}$ HV-CMOS, LFoundry $150 \mathrm{~nm}$ HRCMOS and TowerJazz $180 \mathrm{~nm}$ processes. Prototypes in all processes have been characterized before and after irradiation with thermal neutrons and x-rays.

\section{Recent Results on Pixel Devices with External Readout}

AMS H18 CCPDs The AMS H18 prototypes [3] are small size chips $(\sim 3 \times 4 \mathrm{~mm})$ built on low resistivity $(\sim 10 \Omega \mathrm{cm})$ substrates in multi-project wafer runs. They comprise a two-stage CSA and a discriminator in the pixel cell. Sensors were characterized in laboratory measurements proving radiation tolerance against a TID of up to 1 Grad [4] and showing a strong drift signal after $2 \cdot 10^{16} \mathrm{n}_{\text {eq }} \mathrm{cm}^{-2}[5]$. Testbeam experiments demonstrated a hit detection efficiency above $99.5 \%$ before irradiation [6] which is preserved after irradiation as shown in Figure 1b. A high detection efficiency of $99.7 \%$ was measured in a wide range of threshold settings after a fluence of $1 \cdot 10^{15} \mathrm{neq}_{\mathrm{em}} \mathrm{cm}^{-2}$, which is attributed to a drift based charge collection that also leads to $85 \%$ of the signals being collected in less than $25 \mathrm{~ns}[7]$.

LFoundry 150nm CCPDs These prototypes are produced in an HR-CMOS process on substrates with resistivities above $2 \mathrm{k} \Omega \mathrm{cm}$ and implement, similar to the AMS ones, a CSA and discriminator per pixel cell [8]. The behaviour of the CCPD_LF prototype after irradiation was assessed by charge injection via test pulses and radioactive sources. Figure 2a shows the gain and noise of the 


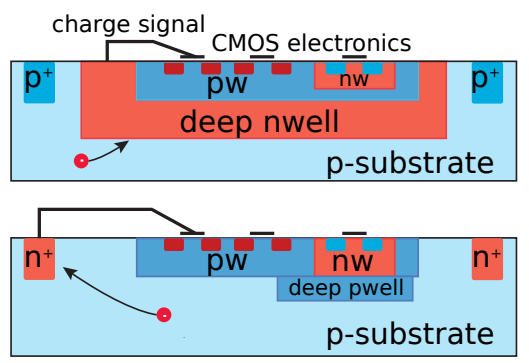

(a)

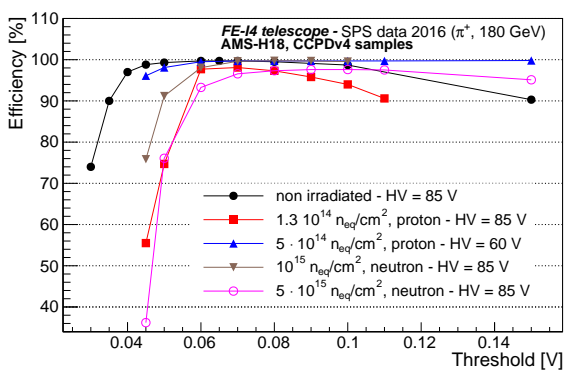

(b)

Fig. 1: (a) Structure of CMOS pixel cells with a large (upper figure) and small (lower figure) fill factor [9]. (b) Hit detection efficiency of AMS H18 prototypes irradiated up to $5 \cdot 10^{15} \mathrm{neq}_{\mathrm{em}}^{-2}[7]$

tested amplifier designs with different feedback transistors after irradiation up to the expected ITk Pixel outer layer TID of 50 Mrad. All prototypes remained functional during the whole irradiation campaign, but showed a decrease in gain of up to $30 \%$. This was tackled with a modified amplifier design in the second generation chip, LF_CPIX, which kept the gain degradation under $10 \%$.

TowerJazz 180nm Investigator In contrast to the formerly discussed prototypes, the TowerJazz Investigator was designed primarily to test different collecting electrode geometries. The small fill factor designs were processed on a $2 \mathrm{k} \Omega \mathrm{cm}$ epitaxial layer with the processing electronics placed in separate deep wells. A lightly doped n-well was implemented as a process modification to allow for a depletion zone under the electronics implants without a significant increase of the diode noise [2]. The pixel cells host a $3 \mathrm{~T}$ (three transistor) processing instead of the CSA and discriminator structure and were read out externally by means of an oscilloscope. Figure 2b shows Sr-90 spectra for a device before and after irradiation to $1 \cdot 10^{15} \mathrm{n}_{\mathrm{eq}} \mathrm{cm}^{-2}$. After voltage to charge calibration using a Fe-55 source no degradation of the signal was found, which was confirmed by a hit detection efficiency of over $98 \%$ measured in testbeam experiments [2].

\section{Monolithic concepts and activities}

The AMS and LFoundry sensors discussed so far were meant as a replacement for current baseline sensors in hybrid detector modules. A more aggressive approach is the development of fully monolithic devices. Depleted Monolithic Active Pixel Sensors (DMAPS) inherently avoid the cost of hybridization and ease detector construction. However, the application of digital circuitry that is compatible with the high trigger rates in ATLAS is challenging in terms of digital crosstalk, speed and power consumption. We are currently investigating several readout 


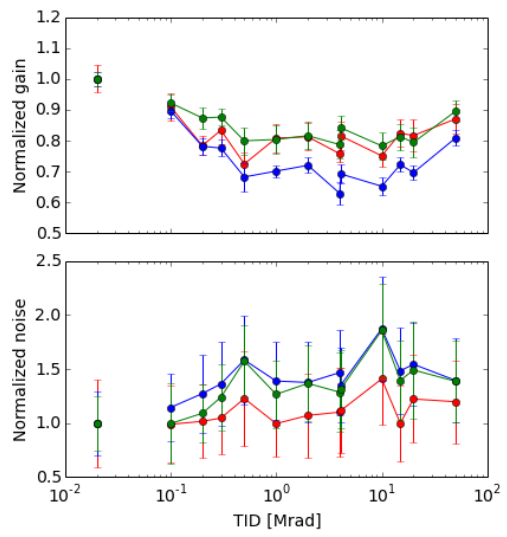

(a)

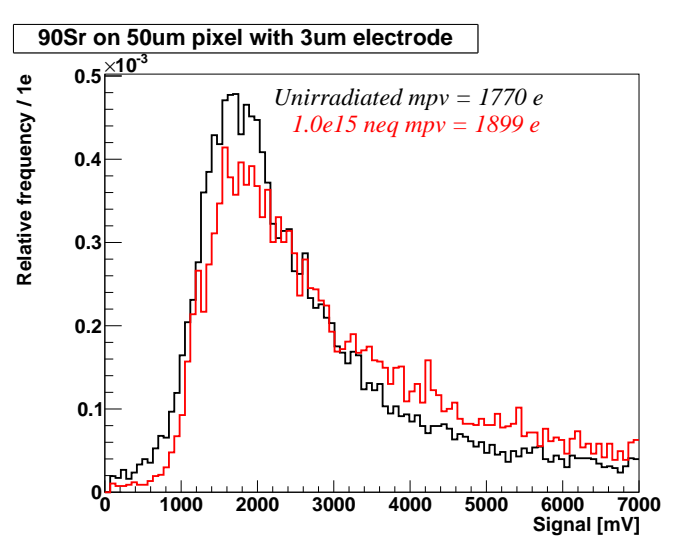

(b)

Fig. 2: (a) Normalized gain and noise of the CCPD_LF prototype amplifiers with default linear transistor (green), long linear transistor (blue) and enclosed layout transistor (red) in the feedback circuit [8]. (b) Single pixel spectrum of a Sr-90 measurement for non-irradiated and $1 \cdot 10^{15} \mathrm{neq}_{\mathrm{em}}^{-2}$ irradiated TowerJazz Investigator pixel cells [10].

strategies implemented in the AMS aH18 (ATLASPIX), the LFoundry $150 \mathrm{~nm}$ (LF-MONOPIX [11]) and the TowerJazz $180 \mathrm{~nm}$ (MALTA) process. The main differences are found in the amount of functionality implemented in the pixel cell and either a triggered or asynchronous readout scheme, which defines the minimally achievable pixel size and digital crosstalk in the matrix.

Implementations of triggered pixel matrices are read out by a column drain architecture (LF-MONOPIX) similar to the current ATLAS Pixel readout chip FE-I3 or using buffer blocks shared by multiple pixels (ATLASPIX) that store hits based on a latency counter (FE-I4 like). The LF-MONOPIX splits into two designs, keeping the necessary readout either in the periphery at the expense of a per pixel connection or in the pixel matrix, shifting that complexity into the pixel cell. The ATLASPIX uses 8-bit wide buses shared by 16 pixels to reduce the complexity of the signal routing, but is only feasible in medium occupancy regions like the outer ITk Pixel layers. A second, triggerless matrix implements one to one connections from the pixel cells to the buffers in the periphery, which immediately pipe out the incoming hit data. Finally, the MALTA chip is an entirely clock-less design where the readout is kept in the pixel cells. Hit information consisting of a pixel group address and hit pattern are sent immediately when the hit is detected via double column and chip wide buses. Collisions are resolved by arbiters that can delay hits for a well defined amount of time on both buses, while charge is measured based on the latency of the hit information compared to an external $40 \mathrm{MHz}$ reference clock. 
A variety of prototypes based on the presented processes or designs is in production or currently under investigation.

\section{Summary}

CMOS based pixel sensors have proven a promising alternative for the outer layers of the ATLAS ITk Pixel Detector. Produced by several foundries they allow for cheap production of large volumes and low-cost interconnection techniques. Recent results showed excellent detection efficiency and speed even after fluences of $1 \cdot 10^{15} \mathrm{neq}_{\mathrm{eq}} \mathrm{cm}^{-2}$.

Following the promising results of the CCPD prototypes an investigation of the possibility of fully monolithic sensors is ongoing. Several designs are in production, implementing different pixel geometries and readout schemes. Characterisation of these sensors will be conducted in 2017 to allow converging on a common design early 2018.

\section{Acknowledgements}

The research leading to these results has received funding from the European Commission under the FP7 Research Infrastructures project AIDA grant agreement no.262025.

\section{References}

1. H. Jansen et al.: Performance of the EUDET-type beam telescopes. EPJTI 2016 3:7

2. H. Pernegger et al.: First tests of a novel radiation hard CMOS sensor process for Depleted Monolithic Active Pixel Sensors. JINST 12(06), P06008 (2017)

3. I. Peric: Active pixel sensors in High-Voltage CMOS technologies for ATLAS. JINST 7(08), C08002 (2012)

4. B. Ristic: Active pixel sensors in ams H18/H35 HV-CMOS technology for the ATLAS HL-LHC upgrade. NIMA 831, 88 - 93 (2016)

5. M. Fernández García et al.: Radiation hardness studies of neutron irradiated CMOS sensors fabricated in the ams H18 high voltage process. JINST 11(02), P02016 (2016)

6. S. Gonzalez-Sevilla et al.: Results of the 2015 testbeam of a $180 \mathrm{~nm}$ ams HighVoltage CMOS sensor prototype. JINST 11(07), P07019 (2016)

7. M. Benoit et al.: Testbeam results of irradiated ams H18 HV-CMOS pixel sensor prototypes. arXiv:1611.02669 (2016)

8. T. Hirono et al.: Characterization of Fully Depleted CMOS Active Pixel Sensors on High Resistive Substrates for Use in a High Radiation Environment. 2016 IEEE NSS MIC conference record, arXiv:1612.03154 (2016)

9. M. Garcia-Sciveres, N. Wermes: Advances in pixel detectors for experiments with high rate and radiation. arXiv:1705.10150 (2017)

10. C. Riegel et al.: Radiation hardness and timing studies of a monolithic TowerJazz pixel design for the new ATLAS Inner Tracker. JINST 12(01), C01015 (2017) 
11. T. Wang et al.: Development of a depleted monolithic CMOS sensor in a $150 \mathrm{~nm}$ CMOS technology for the ATALS Inner Tracker upgrade. JINST 12(01), C01039 (2017) 